\title{
COVID-19 lockdown allows researchers to quantify the effects of human activity on wildlife
}

\author{
Reduced human mobility during the pandemic will reveal critical aspects of our impact on animals, providing \\ important guidance on how best to share space on this crowded planet. \\ Christian Rutz, Matthias-Claudio Loretto, Amanda E. Bates, Sarah C. Davidson, Carlos M. Duarte, \\ Walter Jetz, Mark Johnson, Akiko Kato, Roland Kays, Thomas Mueller, Richard B. Primack, \\ Yan Ropert-Coudert, Marlee A. Tucker, Martin Wikelski and Francesca Cagnacci
}

$\mathrm{O}$ ver the past few months, many countries around the world went into lockdown to control the spread of COVID-19. Brought about by the most tragic circumstances, this period of unusually reduced human mobility - which we suggest be coined 'anthropause' (see Box 1) - may provide important insights into human-wildlife interactions in the twenty-first century. Anecdotal observations indicate that many animal species are enjoying the newly afforded peace and quiet, while others, surprisingly, seem to have come under increased pressure.

Here, we highlight how the international research community can use these extraordinary circumstances to gain unprecedented mechanistic insight into how human activity affects wildlife. We outline urgent steps different stakeholder groups need to take to ensure this opportunity is not missed, and introduce global collaborative research initiatives that are currently forming to facilitate coordination. Scientific knowledge gained during this devastating crisis will allow us to develop innovative strategies for sharing space on this increasingly crowded planet, with benefits for both wildlife and humans.

\section{Possible effects on wildlife}

Social media abound with posts sharing surprising wildlife encounters during lockdown. As we gaze out of our windows, or relish a brief walk in the park, nature appears to have changed, especially in urban environments. There not only seem to be more animals than usual, but there are also some unexpected visitors. People have reported sightings of pumas in downtown Santiago, Chile, of dolphins in untypically calm waters in the harbour of Trieste, Italy, and of jackals in broad daylight in urban parks in Tel Aviv, Israel. Hidden from view, animals may also start roaming more freely across the world's oceans,

\section{Box 1 | Introducing 'anthropause'}

We noticed that people started referring to the lockdown period as the 'Great Pause', but felt that a more precise term would be helpful. We propose 'anthropause' to refer specifically to a considerable global slowing of modern human activities, notably travel. We are aware that the correct prefix is 'anthropo-' (for 'human') but opted for the shortened form, which is easier to remember and use, and where the missing 'po' is still echoed in the pronunciation of 'pause' (po:z).

following reductions in vessel traffic and noise-pollution levels ${ }^{1}$.

But for some species, the pandemic may have created new challenges. For example, various urban-dwelling animals, like rats, gulls or monkeys, have become so reliant on food discarded or provided by humans that they may struggle to make ends meet under current conditions. Interestingly, in some countries where lockdowns allow outdoor exercise, humans are flocking to green spaces in or near metropolitan areas (see Fig. 1), potentially disturbing resident wildlife ${ }^{2}$. At the same time, reduced human presence in more remote areas may potentially expose endangered species, such as rhinos or raptors, to increased risk of poaching or persecution ${ }^{3}$. Finally, concerns have been raised that, in low-income countries, economic hardship may force increased exploitation of natural resources ${ }^{4}$.

At present, it is impossible to say which observations have been hyped by social media, and which expert predictions about global animal responses will hold true. But what is clear is that humans and wildlife have become more interdependent than ever before, and that now is the time to study this complex relationship. A quantitative scientific investigation is urgently needed.

\section{Unprecedented circumstances} As expanding human populations are transforming environments at unprecedented rates, understanding the linkages between human and animal behaviour is of critical importance. It is key to preserving global biodiversity, to maintaining the integrity of ecosystems, and to predicting global zoonoses and environmental change ${ }^{5}$. This knowledge is not only worth billions of dollars, but it is also vital for shaping a sustainable future. So far, however, researchers have had to rely predominantly on purely observational approaches.

Scientists have long sought to quantify how humans impact various aspects of animal biology, such as population levels, reproductive and mortality rates, movement and activity patterns, foraging behaviour, and stress responses ${ }^{1,6-8}$. Studies usually employ one of two main approaches spatial comparisons or temporal analyses. The first involves comparing a species' biology across areas that differ in human activity. Such differences occur, for example, along urban gradients, with increasing distance from coastlines, or between protected and unprotected areas. The second approach documents how animals respond to temporal changes in human activity in a given locality, which may be short-term ${ }^{6}$ (for example, holiday periods, or natural or human-made disasters) or longer-term (for example, changes in protection status, or land- or seascape modification through construction).

The reduction in human mobility on land and at sea during the anthropause is unparalleled in recent history ${ }^{9,10}$. Lockdown effects have been drastic, sudden, and widespread. Countries have also responded in broadly similar ways across large parts of the world, presenting invaluable replicates of this perturbation. So, how exactly can we make the most of these exceptional circumstances? 
While every field study has value in its own right, the pandemic affords an opportunity to build a global picture of animal responses by pooling large numbers of datasets. Such collaborative projects can integrate the spatial and temporal approaches outlined above, in an attempt to uncover causal relationships. Aspects of animals' biology can be compared across sites that vary in COVID-19-related restrictions and resultant changes in human mobility, and across different time periods, spanning from before until after changes occurred. Taking into account additional data from unaffected 'control' sites ${ }^{11}$, such as particularly remote or inaccessible areas, researchers will be able to examine if, and how, animals responded to reductions in human activity. Baseline data from similar time periods in prior years, and from years following the COVID-19 pandemic, will considerably strengthen inferences, helping to disentangle anthropause effects from natural seasonal variation in animal biology.

Finally, we wish to share a very important sentiment. While this is no doubt a valuable research opportunity, it is one that has only come about through tragic circumstances. Scientists who prepare to study lockdown effects on wildlife, and on the environment more generally, should be sensitive to the immense human suffering caused by COVID-19 and use appropriate language to describe their work.

\section{Mobilizing the community}

General insights about animal responses across different species, geographic regions, ecosystems, and levels of human activity - will only be possible if researchers pool their data and expertise. Several initiatives are busy preparing global-scale collaborative research projects to achieve exactly this.

One of them - the COVID-19 Bio-Logging Initiative (www.bio-logging. net) - recently formed under the umbrella of the International Bio-Logging Society, in collaboration with the Movebank research platform, and the Max Planck-Yale Center for Biodiversity Movement and Global Change. This large consortium is planning to use data collected by 'bio-loggers' miniature, animal-attached electronic devices - to measure changes in animals' movement, behaviour, activity and physiology, as well as in the environments they inhabit (see Fig. 1). The project considers all species for which owners are willing to contribute data, and has already received enthusiastic support from both the marine and the terrestrial bio-logging research communities. As their first objective, the team will update an earlier study on terrestrial mammals ${ }^{8}$
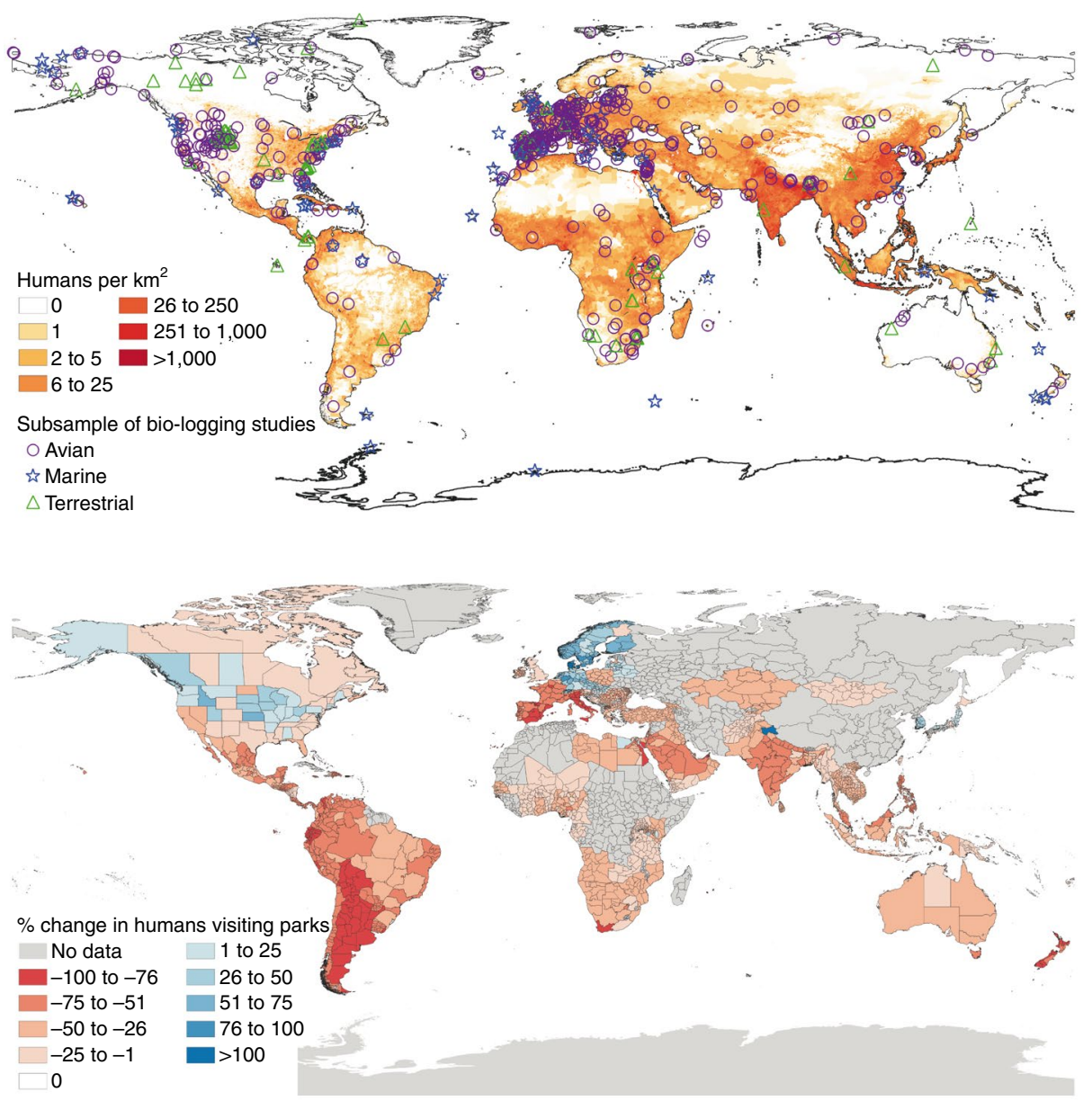

Fig. 1 | Illustrating the research potential of the recently launched COVID-19 Bio-Logging Initiative. Top: locations of a subsample of active animal tracking ('bio-logging') studies superimposed on human population density. Data sources: 801 publicly visible animal tracking studies from the Movebank research platform (www.movebank.org) that are likely to contain data overlapping with the COVID-19 period (data extracted 18 May 2020). 'Marine' includes seabirds and other marine species, 'avian' refers to all other bird species, and 'terrestrial' are non-avian species living mostly on land. Population density data sourced from ref. ${ }^{15}$ (data accessed 15 May 2020). Bottom: median percentage of change based on daily values (with reference to the data provider's default baseline from the five-week period between 3 January and 6 February 2020) in visits to places like local parks, national parks, public beaches, marinas, dog parks, plazas and public gardens for the month of April 2020. Data are plotted for 900 subregions within 131 countries (note that for $1.6 \%$ of the subregions fewer than 5 daily values were available for April 2020). This information should be interpreted cautiously, and is shown here merely to provide a preliminary, coarse-scale illustration of some recent changes in human mobility; scientific analyses will require higher-resolution, calibrated data. Data sourced from ref. ${ }^{16}$ (data accessed 7 May 2020). Both maps were drawn with the QGIS Geographic Information System (http://qgis.org), using freely available data (2018) for country borders from GADM (https://gadm.org) (data accessed 6 May 2020).

with new data from the anthropause, to address a previously intractable question: are the movements of animals in modern landscapes predominantly affected by built structures, or by the presence of humans?

Another initiative - led by the PAN-Environment working group - is planning to assess impacts of human mobility and activity on species and ecosystems by integrating a wide array of information, including data generated by species monitoring programmes, protected area networks, sensor networks and citizen science initiatives. Several additional projects are forming at pace.

These initiatives provide valuable platforms for wildlife biologists, human 
mobility researchers, bioinformaticians and other experts, to join forces for ambitious large-scale analyses. This crisis, and the unique research opportunities it affords, demand such collaboration, as well as full transparency and effective coordination.

\section{Urgent steps}

Immediate action is required from a range of stakeholder groups to ensure that we maximize the scientific insight that arises from this devastating pandemic. Here are some practical recommendations for the short- to mid-term.

First of all, it is of paramount importance that field biologists can continue with data collection even under lockdown conditions ${ }^{2,10,12}$, with appropriate safety precautions. The analyses we outlined above depend on high-quality data, which means a wide range of activities must carry on unhindered, such as instrumenting animals with bio-loggers, servicing of field equipment (for example, camera traps or receiver stations), and conducting routine surveys. Local authorities and research institutions should swiftly issue the required permits ${ }^{12}$.

We are confident that researchers will be keen to resume fieldwork, but recommend they take a few extra steps. First, we suggest they keep detailed records of official restrictions on (and where possible, observed changes in) human mobility in their study areas, as this information may be difficult to reconstruct after the fact. While measures of human activity can be obtained from a variety of 'big data' sources, field observations are required for validation. Second, we encourage the leaders of local projects to get in touch as soon as possible with the larger collaborative initiatives that are being launched, to enable data standardization, exchange of expertise and coordination. Contribution to these initiatives does not preclude independent research outputs, but is essential for global-scale analyses.

Researchers seeking to measure human impact on wildlife often face a frustrating dilemma - they have high-quality data for their study animals, but only crude proxies of human activity. Studies have used land-cover data, proximity to roads or settlements, or fishing vessels' radar signals, to make inferences about human disturbance ${ }^{8}$. These metrics usually offer reasonable approximations, but in situ measurements - such as GPS tracking logs from mobile phones, traffic-flow measurements on land and at sea, and high-resolution satellite images - are required to capture the rapidly changing conditions under lockdown. We urge relevant stakeholders - including wildlife researchers, owners of high-quality human mobility data, experts on data confidentiality, and legislators - to form partnerships that facilitate investigations of anthropause impacts at the highest possible spatio-temporal resolution, in full compliance with the law ${ }^{13}$.

Finally, additional funding is urgently required to support the research programme we envision. This includes funds for field data collection, for data-management infrastructure and support, and for complex data analyses. We know that follow-on field studies are not normally considered a priority by funding agencies, but these are precisely the kinds of projects that can now contribute critically important data series. Field projects must continue data collection during the ups and downs in human mobility we will likely witness over the coming months and beyond.

We do not advocate diverting resources from front-line work or ongoing research on vaccines, diagnostic tests and therapeutics funds for human-wildlife interactions must come from separate parts of governmental budgets that are concerned more broadly with human and environmental health. Some governments have started working on such schemes.

\section{A post-anthropause world}

Society's priority must be to tackle the immense human tragedy and hardship caused by COVID-19. But we cannot afford to miss the opportunity to chart - for the first time on a global scale - the extent to which modern human mobility affects wildlife?

So, what do we hope to learn? Research on anthropause effects will enable a detailed, mechanistic understanding of humanwildlife interactions. It will help us identify species that are seriously affected by human activity, yet still have the capacity to respond to change, as well as others that appear particularly vulnerable. It will also reveal critical thresholds beyond which human disturbance has detrimental effects on animal behaviour, species persistence and ecosystem dynamics, helping us pinpoint processes that negatively feedback on human well-being ${ }^{5}$.

These insights will inspire realistic, evidence-based proposals for improving human-wildlife coexistence. Nobody is asking for humans to remain in a state of permanent lockdown. The COVID-19 anthropause has transported us back to levels of human mobility observed a few decades - not centuries - ago. That means that we may discover that relatively minor changes to our lifestyles can potentially have major benefits for ecosystems and humans. For example, small modifications to the topology and operation of our transport networks may drastically reduce unintended disruptive effects on animal movement.

Coordinated global wildlife research during the anthropause will make contributions that go well beyond informing conservation science - it will challenge humanity to reconsider our future on Earth. There will be unforeseen opportunities to reinvent the way we live our lives, and to forge a mutually beneficial coexistence with other species. It would be wonderful if careful research during this period of crisis helped us to find innovative ways of reining in our increasingly expansive lifestyles, to rediscover how important a healthy environment is for our own well-being, and to replace a sense of owning with a sense of belonging ${ }^{5,14}$. We hope that people will choose to hear the wake-up call.

Christian Rutz (D) 1,2凶,

Matthias-Claudio Loretto (iD) 3,4,

Amanda E. Bates (iD)

Sarah C. Davidson (D) 3,6, Carlos M. Duarte (D)7, Walter Jetz (D) ${ }^{8,9}$, Mark Johnson ${ }^{10,11}$,

Akiko Kato $^{12}$, Roland Kays ${ }^{13,14}$,

Thomas Mueller ${ }^{15,16}$, Richard B. Primack ${ }^{17}$,

Yan Ropert-Coudert (D) 12,

Marlee A. Tucker (D) 18, Martin Wikelski ${ }^{3,6}$ and

Francesca Cagnacci ${ }^{19}$

${ }^{1}$ Centre for Biological Diversity, School of Biology,

University of St Andrews, St Andrews, UK.

${ }^{2}$ Radcliffe Institute for Advanced Study, Harvard

University, Cambridge, MA, USA. ${ }^{3}$ Department

of Migration, Max Planck Institute of Animal

Behavior, Radolfzell, Germany. ${ }^{4}$ Department of

Biology, University of Konstanz, Konstanz, Germany.

${ }^{5}$ Department of Ocean Sciences, Memorial University

of Newfoundland, St. John's, Newfoundland,

Canada. ${ }^{6}$ Centre for the Advanced Study of

Collective Behaviour, University of Konstanz,

Konstanz, Germany. ${ }^{7}$ Red Sea Research Center and

Computational Biosciences Research Center, King

Abdullah University of Science and Technology,

Thuwal, Kingdom of Saudi Arabia. ${ }^{8}$ Ecology and Evolutionary Biology Department, Yale University, New Haven, CT, USA. ${ }^{9}$ Center for Biodiversity and Global Change, Yale University, New Haven, CT, USA. ${ }^{10}$ Sea Mammal Research Unit, University of St Andrews, St Andrews, UK. ${ }^{11}$ Department of Biology, Aarhus University, Aarhus, Denmark. ${ }^{12}$ Centre d'Etudes Biologiques de Chizé, La Rochelle Université - CNRS, UMR 7372, Villiers en Bois, France. ${ }^{13}$ North Carolina Museum of Natural Sciences, Biodiversity Lab, Raleigh, NC, USA. ${ }^{14}$ Fisheries, Wildlife, and Conservation Biology Program, College of Natural Resources, North Carolina State University, Raleigh, NC, USA. ${ }^{15}$ Senckenberg Biodiversity and Climate Research Centre, Senckenberg Gesellschaft für Naturforschung, Frankfurt (Main), Germany. ${ }^{16}$ Department of Biological Sciences, Goethe 
University, Frankfurt (Main), Germany. ${ }^{17}$ Biology Department, Boston University, Boston, MA, USA. ${ }^{18}$ Department of Environmental Science, Institute for Water and Wetland Research, Radboud University, Nijmegen, The Netherlands. ${ }^{19}$ Department of Biodiversity and Molecular Ecology, Research and Innovation Centre, Fondazione Edmund Mach, Trento, Italy.

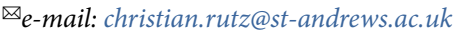

Published online: 22 June 2020

https://doi.org/10.1038/s41559-020-1237-Z

References

1. Jones, N. Nature 568, 158-161 (2019).

2. Corlett, R. T. et al. Biol. Conserv. 246, 108571 (2020).

3. Buckley, R. Biol. Conserv. 247, 108640 (2020).

4. Gardner, C. Nature's comeback? No, the coronavirus pandemic threatens the world's wildlife. The Conversation (14 April 2020).
5. Cook, R. A., Karesh, W. B. \& Osofsky, S. A. The Manhattan Principles on 'One World, One Health' (Wildlife Conservation Society, 2004).

6. Larson, C. L., Reed, S. E., Merenlender, A. M. \& Crooks, K. R. PLOS ONE 11, e0167259 (2016).

7. Gaynor, K. M., Hojnowski, C. E., Carter, N. H. \& Brashares, J. S. Science 360, 1232-1235 (2018)

8. Tucker, M. A. et al. Science 359, 466-469 (2018).

9. Rosenbloom, D. \& Markard, J. Science 368, 447 (2020).

10. Saraswat, R. \& Saraswat, D. A. Science 368, 594-595 (2020).

11. Christie, A. P. et al. J. Appl. Ecol. 56, 2742-2754 (2019).

12. Inouye, D. W., Underwood, N., Inouye, B. D. \& Irwin, R. E. Science 368, 724-725 (2020).

13. Oliver, N. et al. Sci. Adv. 6, eabc0764 (2020).

14. Mace, G. M. Science 345, 1558-1560 (2014).

15. Center For International Earth Science Information Network (CIESIN), Columbia University Gridded Population of the World, version 4 (GPWv4): Population Density Adjusted to Match 2015 Revision UN WPP Country Totals, Revision 11 (2018) (NASA Socioeconomic Data and Applications Center (SEDAC), accessed 15 May 2020); https://doi.org/10.7927/H4F47M65

16. Google COVID-19 Community Mobility Reports (Google LLC, accessed 7 May 2020); https://www.google.com/covid19/mobility/

\section{Acknowledgements}

Manuscript preparation was supported through: a Radcliffe Fellowship at the Radcliffe Institute for Advanced Study, Harvard University (to C.R.); the European Union's Horizon 2020 research and innovation programme under the Marie Skłodowska-Curie grant agreement no. 798091 (to M.-C.L.); and Autonomous Province of Trento ordinary funds to Fondazione Edmund Mach (to F.C.).

Author contributions

The idea for this Comment was conceived by the COVID19 Bio-Logging Initiative. C.R., M.-C.L. and F.C. initiated and coordinated manuscript preparation; C.R. drafted the manuscript with contributions from M.-C.L. and F.C.; M.-C.L. prepared the figure; S.C.D. extracted bio-logging data from Movebank; and all authors provided critical feedback on a draft. Apart from the three lead authors (C.R., M.-C.L. and F.C.), all co-authors are listed alphabetically.

Competing interests

The authors declare no competing interests. 